\title{
Detection of the plasmid-mediated quinolone resistance determinants in clinical isolates of Serratia marcescens in China
}

\author{
Hai-Fei Yang ${ }^{1}$, Jun Cheng ${ }^{1}$, Li-Fen $\mathrm{Hu}^{1}$, Ying Ye ${ }^{1,2,3}$ and Jia-Bin $\mathrm{Li}^{1,2,3}$ \\ The Journal of Antibiotics (2012) 65, 531-533; doi:10.1038/ja.2012.63; published online 1 August 2012
}

Keywords: fluoroquinolones; Qnr; resistance; Serratia marcescens

Serratia marcescens is a prominent opportunistic pathogen responsible for serious infections in immunocompromised individuals, due primarily to its high intrinsic antibiotic resistance. Quinolones are commonly prescribed antimicrobial agents in China, and quinoloneresistant $S$. marcescens isolates have emerged. Yang et al. ${ }^{1}$ reported that the resistance rate of ciprofloxacin and levofloxacin in S. marcescens collected from 15 teaching hospitals located in different areas in China in 2005 was $15.0 \%$ and $7.5 \%$, respectively. Three kinds of plasmid-mediated quinolone resistance (PMQR) determinants have been detected, conferring low-level resistance to quinolones by different mechanisms: quinolone-resistance proteins (Qnr), AAC $\left(6^{\prime}\right)$-Ib-cr, and QepA efflux. ${ }^{2}$ Recently, oqxAB has been identified as another mechanism of PMQR. ${ }^{3}$ As the discovery of Qnr, the presence of different $q n r(\mathrm{~A}, \mathrm{~B}, \mathrm{~S}, \mathrm{C}$ and $\mathrm{D})$ in plasmids has been found worldwide in different bacterial pathogens. ${ }^{4,5}$ Resistance to quinolones can also be caused by mutations in the chromosomal genes that code for DNA gyrase and/or DNA topoisomerase IV. ${ }^{6}$ Fujimaki $\mathrm{K}$ et al. ${ }^{7}$ reported that DNA gyrase alterations are the basis of quinolone resistance in clinical isolates of $S$. marcescens. Watanabe $\mathrm{M}$ et al. ${ }^{8}$ showed that the mutation in DNA gyrase and mutations that appear to decrease drug permeation occurred frequently in quinolone-resistant isolates of $S$. marcescens. It has been found that the amino-acid residues most frequently mutated in GyrA from $S$. marcescens occur at Gly-81, Ser-83 and Asp-87. Park et al. ${ }^{9}$ examined 166 isolates of $S$. marcescens and reported one positive for qnrA and three for $q n r B$. We conducted this study to estimate the prevalence of PMQR determinants and the role of mutations in DNA gyrase and/or DNA topoisomerase IV in S. marcescens in Anhui, China.

A total of 146 nonduplicate $S$. marcescens isolates were collected in 34 hospitals from 2005 to 2011 in Anhui, China. Species identification was performed with the Vitek 2 system (bioMérieux, Marcy l' Étoile, France) and confirmed with API 20E (bioMérieux). The minimum inhibitory concentrations (MICs) for ciprofloxacin, levofloxacin and gatifloxacin (Oxoid) were further determined by the agar dilution method in accordance with the recommendations of the Clinical Laboratory Standards Institute (CLSI, 2012). ${ }^{10}$ All isolates were screened for the presence of qnrA, qnrB, qnrS by multiplex PCR using the primers as Robicsek et al. ${ }^{11}$ as described. Amplification products were provisionally identified from their sizes in ethidium bromide-stained agarose gels. Positive results were confirmed by amplification with primers $5^{\prime}$-ATGACGCCATTACTGTATAA- $3^{\prime}$ and $5^{\prime}$-GATCGCAATGTGTGAAGTTT-3' for $q n r B .^{12} a a c\left(6^{\prime}\right)-I b$ was amplified by PCR as Park et al. ${ }^{12}$ described. All positives were further analyzed by direct sequencing of the PCR products with primer $5^{\prime}$-CGTCACTCCATACATTGCAA- $3^{\prime}$ to identify aac $\left(6^{\prime}\right)$-Ib-cr. $q n r C, q n r D$, qеpA, and $o q x A$ genes were screened by using the primers as described previously. ${ }^{3,13-15}$ The gyrA and gyrB genes of DNA gyrase and the parC and parE genes of topoisomerase IV were amplified by PCR using the primers, as previously described. ${ }^{16}$ All the purified PCR products were sequenced on an ABI PRISM3730 sequencer analyzer (Applied Biosystems, Foster City, CA, USA). The nucleotide and deduced protein sequences were analyzed with software available from the National Center for Biotechnology Information. Conjugation experiments were carried out in Luria-Bertani (LB) broth with sodium azide-resistant Escherichia coli J53 as the recipient, as previously described. ${ }^{17}$ Transconjugants were selected on LB agar plates supplemented with sodium azide $\left(100 \mu \mathrm{g} \mathrm{ml}^{-1}\right)$ (Sigma Chemical Co., St Louis, MO, USA) and ciprofloxacin $\left(0.25 \mu \mathrm{g} \mathrm{ml}^{-1}\right)$.

Among the $146 \mathrm{~S}$. marcescens isolates, the total resistance rates to ciprofloxacin, levofloxacin and gatifloxacin were $27.4 \%$ (40/146), $17.1 \%(25 / 146)$, and $15.8 \%$ (23/146), respectively. The quinolone resistance rates for all isolates per year were shown in Table 1. The quinolone resistance rates in our study were higher than the reports of Yang et al., ${ }^{1}$ which shown that the resistance rate of ciprofloxacin and levofloxacin in S. marcescens collected from 15 teaching hospitals in China in 2005 was $15.0 \%$ and $7.5 \%$, respectively. The differences may

${ }^{1}$ Department of Infectious Disease, the First Affiliated Hospital of Anhui Medical University, Hefei, China; ${ }^{2}$ Institute of Bacterium Resistance, Anhui Medical University, Hefei, China and ${ }^{3}$ Anhui Center for Surveillance of Bacterial Resistance, Hefei, China

Correspondence: Dr J-B Li, Department of Infectious Disease, the First Affiliated Hospital of Anhui Medical University, Hefei 230022, China.

E-mail: lijiabin948@vip.sohu.com

Received 15 March 2012; revised 24 May 2012; accepted 26 June 2012; published online 1 August 2012 
Table 1 The quinolone resistance rates in S. marcescens during recent 7 years in China

\begin{tabular}{lccccrrr}
\hline & \multicolumn{9}{c}{ Years } \\
\cline { 2 - 7 } & $2005(\mathrm{~N}=14)^{\mathrm{a}}$ & $2006(\mathrm{~N}=30)$ & $2007(\mathrm{~N}=16)$ & $2008(\mathrm{~N}=22)$ & $2009(\mathrm{~N}=15)$ & $2010(\mathrm{~N}=16)$ & $2011(\mathrm{~N}=33)$ \\
Quinolones & $R \%(\mathrm{n})^{\mathrm{b}}$ & $R \%(\mathrm{n})$ & $R \%(\mathrm{n})$ & $R \%(\mathrm{n})$ & $R \%(\mathrm{n})$ & $R \%(\mathrm{n})$ & $R \%(\mathrm{n})$ \\
\hline Ciprofloxacin & $42.9(6)$ & $33.3(10)$ & $12.5(2)$ & $18.2(4)$ & $26.7(4)$ & $31.3(5)$ & $27.3(9)$ \\
Levofloxacin & $28.6(4)$ & $16.7(5)$ & $6.25(1)$ & $9.1(2)$ & $20.0(3)$ & $31.3(5)$ & $15.2(5)$ \\
Gatifloxacin & $14.3(2)$ & $16.7(5)$ & $6.25(1)$ & $4.5(1)$ & $26.7(4)$ & $12.5(2)$ & $24.2(8)$ \\
\hline
\end{tabular}

${ }^{a} N=$ the total number of isolates in that year.

${ }^{\mathrm{b}} \mathrm{R} \%=$ the resistance rates; $n=$ the number of resistant isolates.

Table 2 PMQR determinants and alterations in gyrA and parC in S. marcescens isolates

\begin{tabular}{|c|c|c|c|c|c|c|c|c|}
\hline \multirow[b]{3}{*}{ Strain } & \multirow[b]{3}{*}{ Year } & \multicolumn{3}{|c|}{$\operatorname{MICs}\left(\mu g m I^{-1}\right)$} & \multirow[b]{3}{*}{$P M Q R$ determinants } & \multicolumn{3}{|c|}{ Nucleotide and amino-acid change } \\
\hline & & \multirow[b]{2}{*}{$C I P^{a}$} & \multirow[b]{2}{*}{$L V X^{a}$} & \multirow[b]{2}{*}{$G A T^{\mathrm{a}}$} & & \multicolumn{2}{|c|}{ gyrA } & \multirow{2}{*}{$\begin{array}{c}\text { parC } \\
\text { Ser80 (AGC) }\end{array}$} \\
\hline & & & & & & Ser83 (TCG) & Asp87 (GAC) & \\
\hline 9 & 2005 & 16 & 8 & 4 & $a a c\left(6^{\prime}\right)-I b-c r$ & Leu (TTG) & - & Ile (ATC) \\
\hline 12 & 2005 & $>32$ & 32 & 16 & $\operatorname{aac}\left(6^{\prime}\right)-I b-c r, q n r S 2$ & Leu (TTG) & Asn (AAC) & Ile (ATC) \\
\hline $\mathrm{T} 12^{\mathrm{b}}$ & & 2 & 2 & 2 & $\operatorname{aac}\left(6^{\prime}\right)-I b-c r, q n r S 2$ & - & - & - \\
\hline 22 & 2006 & 32 & 16 & 16 & $q n r B 6$ & Leu (TTG) & Asn (AAC) & - \\
\hline $\mathrm{T} 22^{\mathrm{b}}$ & & 1 & 0.25 & 0.25 & qnrB6 & - & - & - \\
\hline 60 & 2007 & 16 & 16 & 8 & $\operatorname{aac}\left(6^{\prime}\right)-1 b-c r$ & - & - & Ile (ATC) \\
\hline $\mathrm{T} 60^{\mathrm{b}}$ & & 0.5 & 0.25 & 0.25 & $\operatorname{aac}\left(6^{\prime}\right)-I b-c r$ & - & - & - \\
\hline 64 & 2008 & 32 & 32 & 8 & qnrS2 & Leu (TTG) & Asn (AAC) & - \\
\hline $\mathrm{T} 64^{\mathrm{b}}$ & & 1 & 0.25 & 0.25 & qnrS2 & - & - & - \\
\hline 86 & 2009 & $>32$ & 32 & 32 & $\operatorname{aac}\left(6^{\prime}\right)-I b-c r$ & Leu (TTG) & Asn (AAC) & Ile (ATT) \\
\hline T86 & & 0.5 & 0.25 & 0.25 & $\operatorname{aac}\left(6^{\prime}\right)-I b-c r$ & - & - & - \\
\hline 91 & 2009 & $>32$ & 32 & 32 & $\operatorname{aac}\left(6^{\prime}\right)-I b-c r, q n r B 6$ & Leu (TTG) & - & Ile (ATC) \\
\hline T91 & & 2 & 1 & 1 & $\operatorname{aac}\left(6^{\prime}\right)-I b-c r$, qnrB6 & - & - & - \\
\hline$J 53 A Z^{R ~ c}$ & & $<0.0625$ & $<0.125$ & $<0.125$ & $-^{d}$ & - & - & - \\
\hline
\end{tabular}

Abbreviations: CIP, Ciprofloxacin; GAT, Gatifloxacin; LVX, Levofloxacin; MICs, minimum inhibitory concentrations; PMQR, plasmid-mediated quinolone resistance.

aThe resistance breakpoints for $\mathrm{CIP}$, LVX and GAT are $\geqslant 4, \geqslant 8$, and $\geqslant 8$, respectively.

aThe resistance breakpoint
${ }^{\mathrm{b}} \mathrm{T}=$ transconjugant of.

'Sodium azide-resistant Escherichia coli J53.

${ }^{\mathrm{d}}$ No alterations and genes.

be due to the extensive use of quinolone in clinic condition in recent years in China. PMQR determinants were present in seven $(4.8 \%)$ isolates with $q n r$ and $a a c\left(6^{\prime}\right)-I b-c r$ detected alone or in combination. Mutations in $g y r A$ and/or parC were identified in those seven PMQRpositive isolates simultaneously (Table 2). Those seven PMQRpositive isolates were all resistant to fluoroquinolones according to CLSI 2012. The most frequently found gene was $a a c\left(6^{\prime}\right)-I b-c r$, which was identified in $5(3.4 \%)$ isolates. Two $a a c\left(6^{\prime}\right)$ - Ib-cr-positive isolates carried $q n r S 2$ and $q n r B 6$, respectively. This agrees with the previous reports that $q n r$ alleles were coexpressed with $a a c\left(6^{\prime}\right)-I b-c r$ on the same plasmid. ${ }^{18} q n r S$ and $q n r B$ were identified in two isolates, respectively. However, other PMQR genes ( $q n r A$, qnrC, qnrD, qepA and $o q \times A$ ) were not found in these isolates. Kim SY et al. ${ }^{19}$ observed significant association of $a a c\left(6^{\prime}\right)-I b-c r$ with $q n r A$ and $q n r S$ in a Korean study. And most isolates with both $a a c\left(6^{\prime}\right)-I b-c r$ and $q n r$ genes showed higher levels of quinolone resistance than those with $\operatorname{aac}\left(6^{\prime}\right)-I b-c r$ alone.

Mutations in the quinolone resistance-determining regions of gyrA and/or parC were identified amongst the seven PMQR-positive isolates, whereas no gyrB or parE mutations were found. The most common mutation found in PMQR-positive isolates was at codon 83 of $g y r A$ (TCG $\rightarrow$ TTG transition), resulting in the replacement of serine by leucine. Four isolates were found with a combination of a mutation in parC at codon 80 and a mutation in the gyrA at codon 83 or codon 87. The mutations of parC, plus other gyrA mutations, conferred a higher level of resistance to fluoroquinolones than the mutation at codon 80 of parC in the study. Earlier studies have described alterations in DNA gyrase in quinolone-resistant S. marcescens isolates but did not examine the topoisomerase IV genes. $^{20,21}$ Weigel LM et al. ${ }^{21}$ reported that the fluoroquinoloneresistant clinical isolates of $S$. marcescens displayed the greatest diversity in mutations, including Gly-81 to Cys, Ser-83 to Ile or Arg and Asp-87 to Asn. Interestingly, in contrast with other enterobacterial species, mutation of Ser- 83 was not required for high-level fluoroquinolone resistance in S. marcescens. Kim JH et al. ${ }^{20}$ reported that the Ser-83-to-Arg substitution in GyrA protein might lead to high-level quinolone resistance in $S$. marcescens by introducing a bulky amino-acid residue into the protein and also by decreasing the hydrogen-bonding capacity between amino-acid residues. In our study, the mutation at codon $83(\mathrm{C} \rightarrow \mathrm{T}$ transition $)$ in $g y r A$ has also 
been detected, resulting in the replacement of serine (TCG) by leucine (TTG).

Among the seven PMQR-positive isolates, plasmids from six of the isolates were successfully transferred to the recipients, suggesting that the dissemination of the PMQR determinants is mostly due to the transmission of plasmids by horizontal exchange. An increase in the MICs of quinolones was detected in the transconjugants compared with the recipients (Table 2).

Our study described clinical isolates of $S$. marcescens in China carrying plasmid-mediated quinolone-resistant genes (qnrB6, qnrS2 and/or $\left.a a c\left(6^{\prime}\right)-I b-c r\right)$ together with mutations in gyrA and parC genes. These mechanisms were likely to have contributed individually to the high level of ciprofloxacin, levofloxacin and gatifloxacin resistance in S. marcescens.

\section{NUCLEOTIDE SEQUENCE ACCESSION NUMBERS}

The sequences of the qnrB6, qnrS2 and $a a c\left(6^{\prime}\right)-I b-c r$ reported in this article have been deposited in the GenBank database and assigned accession numbers JQ034317, JQ041635 and JQ034318, respectively. gyrA and parC genes have also been deposited in GenBank under accession number JQ034320 and JQ235843 for gyrA, JQ235844 for parC.

\section{CONFLICT OF INTEREST}

The authors declare no conflict of interest.

\section{ACKNOWLEDGEMENTS}

We thank all the contributing hospitals that provided isolates for this study. This work was supported by grants from the National Natural Science Foundation of China (no. 30972631and no. 81101288) and the Natural Science Foundation of Anhui Province, China (no. 11040606Q23).

1 Yang, Q. W. et al. Surveillance of antimicrobial resistance among nosocomial gramnegative pathogens from 15 teaching hospitals in China in 2005. Nat.l Med. J. China 87, 2753-2758 (2007) [in Chinese].

2 Robicsek, A., Jacoby, G. A. \& Hooper, D. C. The worldwide emergence of plasmidmediated quinolone resistance. Lancet Infect. Dis. 6, 629-640 (2006)

$3 \mathrm{Kim}, \mathrm{H}$. B. et al. oqxAB encoding a multidrug efflux pump in human clinical isolates of Enterobacteriaceae. Antimicrob. Agents Chemother. 53, 3582-3584 (2009).

4 Martínez-Martínez, L. et al. Plasmid-mediated quinolone resistance. Expert Rev. Anti Infect. Ther. 182, 685-711 (2008).
5 Cattoir, V., Poirel, L., Mazel, D., Soussy, C. J. \& Nordmann, P. Vibrio splendidus as the source of plasmid-mediated QnrS-like quinolone resistance determinants. Antimicrob. Agents Chemother. 51, 2650-2651 (2007).

6 Ruiz, J. Mechanisms of resistance to quinolones: target alterations, decreased accumulation and DNA gyrase protection. J. Antimicrob. Chemother. 51, 1109-1117 (2003).

7 Fujimaki, K. et al. Quinolone resistance in clinical isolates of Serratia marcescens. Antimicrob. Agents Chemother. 33, 785-787 (1989).

8 Watanabe, M., Kotera, Y., Yosue, K., Inoue, M. \& Mitsuhashi, S. In vitro emergence of quinolone-resistant mutants of Escherichia coli, Enterobacter cloacae, and Serratia marcescens. Antimicrob. Agents Chemother. 34, 173-175 (1990).

9 Park, Y. J., Yu, J. K., Lee, S., Oh, E. J. \& Woo, G. J. Prevalence and diversity of $q n r$ alleles in AmpC-producing Enterobacter cloacae, Enterobacter aerogenes, Citrobacter freundii and Serratia marcescens: a multicentre study from Korea. J. Antimicrob. Chemother. 60, 868-871 (2007).

10 Clinical and Laboratory Standards Institute. Performance standards for antimicrobial susceptibility testing. Twenty-Second informational supplement M100-S22. Clinical and Laboratory Standards Institute, Wayne, PA, USA (2012).

11 Robicsek, A., Strahilevitz, J., Sahm, D. F., Jacoby, G. A. \& Hooper, D. C. qnr Prevalence in ceftazidime-resistant enterobacteriaceae isolates from the United States. Antimicrob. Agents Chemother. 50, 2872-2874 (2006).

12 Park, C. H., Robicsek, A., Jacoby, G. A., Sahm, D. \& Hooper, D. C. Prevalence in the United States of $\operatorname{aac}\left(6^{\prime}\right)-I b$-cr encoding a ciprofloxacin-modifying enzyme. Antimicrob. Agents Chemother. 50, 3953-3955 (2006).

13 Cavaco, L. M., Hasman, H., Xia, S. \& Aarestrup, F. M. QnrD a novel gene confering transferable quinolone resistance in Salmonella enterica serovar Kentucky and Bovismorbificans strains of human origin. Antimicrob. Agents Chemother. 53, 603-608 (2009).

14 Wang, M. et al. New plasmid-mediated quinolone resistance gene, qnrC, found in a clinical isolate of Proteus mirabilis. Antimicrob. Agents Chemother. 53, 1892-1897 (2009).

15 Yamane, K., Wachino, J. \& Suzuki, S. New plasmid-mediated fluoroquinolone efflux pump, QepA, found in an Escherichia coli clinical isolate. Antimicrob. Agents Chemother. 51, 3354-3360 (2007).

16 Dutta, S., Kawamura, Y. \& Ezaki, T. Alteration in the GyrA subunit of DNA gyrase and the ParC subunit of topoisomerase IV in quinolone-resistant Shigella dysenteriae serotype I clinical isolates from Kolkata, India. Antimicrob. Agents Chemother. 49, 1660-1661 (2005).

17 Wang, M. et al. Plasmid-mediated quinolone resistance in clinical isolates of Escherichia coli from Shanghai, China. Antimicrob. Agents Chemother. 47, 2242-2248 (2003)

18 Jiang, Y. et al. Plasmid-mediated quinolone resistance determinants $q h r$ and $\operatorname{aac}\left(6^{\prime}\right)$ $\mathrm{Ib}$-cr in extended-spectrum $\beta$-lactamase-producing Escherichia coli and Klebsiella pneumoniae in China. J. Antimicrob. Chemother. 61, 1003-1006 (2008).

19 Kim, S. Y., Park, Y. J., Yu, J. K., Kim, Y. S. \& Han, K. Prevalence and characteristics of aac (6')-Ib-cr in AmpC-producing Enterobacter cloacae, Citrobacter freundii, and Serratia marcescens: a multicenter study from Korea. Diagn. Microbiol. Infect. Dis. 63, 314-318 (2009)

$20 \mathrm{Kim}$, J. H., Cho, E. H., Kim, K. S., Kim, H. Y. \& Kim, Y. M. Cloning and nucleotide sequence of the DNA gyrase gyrA gene from Serratia marcescens and characterization of mutations in gyrA of quinolone-resistant clinical isolates. Antimicrob. Agents Chemother. 42, 190-193 (1998).

21 Weigel, L. M., Steward, C. D. \& Tenover, F. C. gyrA mutations associated with fluoroquinolone resistance in eight species of Enterobacteriaceae. Antimicrob. Agents Chemother. 42, 2661-2667 (1998). 